\title{
Determinantes sociales de salud en los agricultores del resguardo indigena Zenú
}

\author{
Social determinants of health in indigenous farmers \\ of shelter Zenú
}

Elsy C. Puello A. ${ }^{1}$, Concepción E. Amador A. ${ }^{1}$, José M. Luna R. ${ }^{1}$

Forma de citar: Puello EC, Amador CE, Luna JM. Determinantes sociales de salud en los agricultores del resguardo indígena Zenú. Rev Univ Ind Santander Salud. 2016; 48(1): 17-26. DOI: http://dx.doi.org/10.18273/revsal.v48n1-

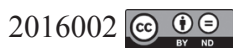

\section{RESUMEN}

Objetivo: Analizar los determinantes sociales de salud presentes entre los indígenas agricultores del Resguardo Zenú de San Andrés de Sotavento. Metodología: Estudio descriptivo de corte, con enfoque cuantitativo, participaron 64 indígenas agricultores de las comunidades Celeste Imperio y Santa Elena del Municipio de San Andrés de Sotavento en el año 2012, se seleccionaron bajo la técnica del muestreo aleatorio simple. La recolección de la información se efectuó a través de encuestas estructuradas diligenciadas mediante entrevista. Resultados: El rango de edad de la población sujeto osciló entre 18 a 70 años, existe $41 \%$ de analfabetismo absoluto, las familias son extensas con orientación endogámica, las condiciones de vida son precarias, hay ausencia de actividad recreativa y actividad física programada, 97\% percibe menos de un salario mínimo mensual legal vigente producto del trabajo comunitario, $95.3 \%$ pertenece al regimen de salud subsidiado, todos carecen de afiliación a riesgos laborales y pensión, $77 \%$ admitió que tuvo algún accidente laboral en el último año, así mismo, 70\% manifestó morbilidad sentida relacionada con la actividad laboral. Conclusiones: Los determinantes de carácter social que influyen negativamente en la salud identificados fueron: sociopolítico, circunstancias materiales, factores biológicos, conductuales y laborales; los cuales se reflejan en deficiencias en su calidad de vida, morbilidad sentida, accidentalidad laboral, fragilidades en el Sistema de Protección Social, altos índices de analfabetismo absoluto, pobreza y características que indiscutiblemente reafirman la relación perversa e interdependiente entre analfabetismo, pobreza y salud. Sin embargo, la cohesión social se identificó como determinante social de influencia positiva para la salud (94\%).

Palabras claves: Indígenas agricultores, escolaridad, pobreza, determinantes de salud.

1. Universidad de Córdoba. Montería, Colombia.

Correspondencia: Elsy C. Puello A. Dirección: Carrera 8 №64a-31. Montería. Email. elsypuello@hotmail.com Teléfono: 3205312947

Recibido: Noviembre 19 de 2014 Aprobado: Agosto 24 de 2015 


\section{ABSTRACT}

Objective: To analyze the social determinants of health present among indigenous farmers of Zenú reservation of San Andrés de Sotavento. Methodology: Descriptive cutting with a quantitative approach, 64 indigenous farmers of communities Celeste Imperio and Santa Elena of the municipality of San Andrés de Sotavento participated in 2012; they were selected by simple random sampling technique. The data collection was conducted through structured surveys filled by interview. Results: The age range of the target population oscillated from 18 to 70 years, there is $41 \%$ of total illiteracy, families are large with inbred orientation, living conditions are poor, there are no recreational activity or physical activity programs, $97 \%$ earn less than the monthly legal minimum wage of community work product, $95.3 \%$ belongs to the subsidized health regime, all lack of affiliation to occupational hazards and pension, $77 \%$ admitted that they had some accident in the last year also $70 \%$ said they felt morbidity related to work activity. Conclusions: The identified social determinants that negatively affect health were: sociopolitical, material circumstances, biological, behavioral and occupational factors; which are reflected in deficiencies in their quality of life, perceived morbidity, occupational accidents, weaknesses in the social protection system, high levels of absolute illiteracy, poverty and characteristics that undoubtedly reinforce the perverse and interdependent relationship between illiteracy, poverty and health. However, social cohesion was identified as a positive influence of health $(94 \%)$.

Keywords: Indigenous farmers, education, poverty, health determinants.

\section{INTRODUCCIÓN}

La Organización Mundial de la Salud, ha definido los determinantes sociales de la salud como aquellas "circunstancias en que las personas nacen, crecen, viven, trabajan y envejecen, incluido el sistema de salud. Esas circunstancias son el resultado de la distribución del dinero, el poder y los recursos a nivel mundial, nacional y local, que depende a su vez de las políticas adoptadas"'.

En el marco de las observaciones anteriores, puede señalarse que la situación de salud de los pueblos indígenas se deteriora cuando sus oportunidades son restringidas, situación que empeora en la medida en que el analfabetismo y la pobreza aumentan; estableciéndose una relación categórica negativa entre analfabetismo, pobreza y salud. En este sentido, disminuir la pobreza y el analfabetismo absoluto (entendido como no saber leer ni escribir) contribuiría en forma decisiva a aumentar significativamente la salud y el desarrollo de este grupo étnico, porque al mejorar su desarrollo cognoscitivo, se pueden ampliar sus posibilidades de acceso a la información y de incrementar la capacidad de autocuidado personal, familiar y laboral.

Históricamente, los pueblos indígenas han contribuido a la protección del patrimonio cultural, histórico, lingüístico y ambiental de la población mundial, no obstante, pese a sus invaluables aportes, estudiosos del tema afirman que existe una profunda desigualdad de oportunidades que ha caracterizado a estos grupos étnicos y continúan siendo vulnerables en diversos aspectos: sociales, económicos y políticos, hasta tal punto que sus condiciones de salud, pobreza, analfabetismo, generación de ingresos, protección social y acceso a servicios son más precarias en ellos que en otros grupos poblacionales ${ }^{2-6}$.

Sin embargo, a pesar de las adversidades, las pocas oportunidades y los procesos de eliminación e invisibilización a los que ha sido sometido este grupo poblacional, su energía, valor, paciencia, lucha y resistencia reafirman su rol como actores imprescindibles en las acciones relacionadas con el mejoramiento de la salud en nuestro continente ${ }^{7}$.

Los resultados que se presentan en este artículo tienen gran validez contextual sobre todo si se considera que los entornos hogareños y comunitarios son los ambientes donde mejor se valoran los aspectos culturales de un grupo social, y estos son valiosos porque el contexto geográfico donde se desarrollan las personas es un marcador por excelencia del carácter de los habitantes de una región y éste a su vez, es un indicador del comportamiento en los asuntos relativos a la salud ${ }^{8}$.

Desde esta perspectiva, para el equipo interdisciplinario el conocimiento de los determinantes sociales de la salud en la cultura Zenú cobra gran importancia. Para la enfermería en particular, los hallazgos son fundamentales para brindar un cuidado profesional en promoción y prevención acorde con las necesidades reales del contexto de la población analizada y para la agronomía representan un insumo para comprender las situaciones que afectan el desarrollo rural y los niveles de productividad agrícola de estas comunidades. 
De igual manera, a nivel general contribuiría para que las autoridades nacionales, regionales, locales y las diversas comunidades tengan una mayor comprensión de los determinantes sociales de salud en los agricultores del resguardo indígena Zenú; partiendo de conocimiento y vivencias de esta comunidad, de tal forma que posteriormente existan elementos conceptuales que permitan a las autoridades competentes en un futuro mediato tomar las medidas concernientes con relación a los problemas derivados de esta realidad, así como elaborar, ajustar y/o retomar políticas que beneficien la calidad de vida de estos colectivos.

Es necesario esclarecer que el gobierno colombiano ha realizado avances en la búsqueda del equilibrio con los sectores poblacionales minoritarios, estableciendo prioridades y determinando que toda la población étnica se encuentra en condición de vulnerabilidad, situación que permite el acceso a bienes y servicios con facilidades legales respetando los principios culturales, raciales y sociales propios de cada etnia; estas condiciones facilitan las posibilidades de autonomía y desarrollo de la población indígena, no obstante en lo relacionado con la escolarización, la promoción y el mantenimiento de la salud de estos pueblos, los esfuerzos no han sido suficientes o acordes a la magnitud del problema, por los elevados índices de analfabetismo, que a su vez se reflejan en deficiente ingreso económico y precarias condiciones de vida.

En este orden de ideas, puede apreciarse que la salud de las personas depende de la compleja relación y de las interconexiones de una serie de determinantes sociales y económicos, dentro de los cuales cobran un papel protagónico y de gran trascendencia la pobreza, el analfabetismo, la desnutrición, el desempleo, condiciones de género y etnia. Elementos señalados a ser intervenidos de forma perentoria por los Objetivos de Desarrollo del Milenio (ODM), precisamente para poder reducirlos al considerar que los determinantes sociales de la salud explican la mayor parte de las inequidades sanitarias.

Los ODM tienen una visión de desarrollo que privilegia la salud y la educación así como la preservación del entorno como motor del desarrollo, es tal la importancia de los determinantes de la salud en los pueblos, que los objetivos, metas e indicadores establecidos para la consecución de los ODM tienen una relación directa con ella y están enfocados a la consecución de la misma particularizando en los grupos más vulnerables ${ }^{9}$ entre los cuales se encuentran las comunidades indígenas.
Sin lugar a dudas, el análisis de todas las dimensiones inherentes a la labor agrícola de la población indígena reviste gran relevancia, no obstante, para efectos de este artículo sólo se analizarán los determinantes sociales de salud presentes en algunas comunidades agricultoras del Resguardo Zenú de San Andrés de Sotavento, conscientes que la identificación y análisis de estos determinantes de salud, evidencia el grado de desprotección en el que se encuentra estos colectivos humanos, situación que genera pobreza, exclusión y desigualdad social.

De acuerdo con los razonamientos que se han venido señalando, el estudio, se realizó con el objetivo de establecer las formas como la escolaridad y los ingresos económicos actúan como determinantes sociales de salud de los indígenas agricultores del Resguardo Zenú de San Andrés de Sotavento, así mismo, evidenciar ante los organismos gubernamentales y no gubernamentales, la situación real de desprotección social y pobreza en la que aún se encuentra rezagada esta población colombiana.

\section{METODOLOGÍA}

Se realizó un estudio descriptivo, de corte, con enfoque cuantitativo, desarrollado en el municipio de San Andrés de Sotavento, específicamente en las comunidades rurales de Celeste Imperio y Santa Elena, pertenecientes al Resguardo Indígena Zenú.

La población estuvo conformada por 572 personas, distribuidas en 90 familias que habitan en los predios del territorio colectivo ${ }^{10}$ cuya actividad económica se basa en la agricultura familiar y comunitaria, por tanto la unidad de análisis la constituyó el indígena agricultor. Se efectuó un muestreo probabilístico, tipo aleatorio simple donde cada persona tuvo la posibilidad de pertenecer a la muestra. Para cálculo del tamaño de muestra se utilizó el programa STATS ${ }^{\mathrm{TM}}$ con nivel de confianza de $95 \%$, porcentaje estimado de la muestra de $50 \%$ y porcentaje de error del $5 \%$. La muestra estuvo constituida por 64 agricultores, por respeto a la condición de menor trabajador sólo se incluyeron los mayores de 17 años.

Para asegurar los principios de participación voluntaria, el consentimiento libre, previo e informado escrito, se contactó a la Capitana del Resguardo Indígena y a algunos líderes, posteriormente se informó a toda la población para motivar a quienes de manera voluntaria quisieran participar. Los datos se recolectaron durante los meses de marzo a junio de 2012. En la encuesta se agrupan cuatro aspectos de información: socio 
demográfica, condiciones laborales, percepción sobre riesgos ocupacionales y la relacionada con los determinantes sociales que influyen en la salud (contexto sociopolítico, circunstancias materiales, factores biológicos incluidas las condiciones sociales de los primeros años de la vida, así como aspectos de los factores conductuales y de cohesión que tienen su expresión como determinante social, pero, para efectos de este artículo, sólo se presentarán los datos concernientes a los determinantes sociales de salud.

Una vez obtenida la información, ésta se procesó en Excel versión 2007 y se analizó utilizando la estadística descriptiva. Los datos se presentan en tablas y gráficos en orden de los objetivos establecidos.

Los aspectos éticos y legales manejados en la investigación se fundamentaron en la Resolución 008430 de 1993 la cual establece las bases conceptuales y logros de la investigación en salud. El estudio se clasificó como "sin riesgo ético". A cada participante se le dio a conocer los objetivos para lograr su participación a través del consentimiento informado.

\section{RESULTADOS}

Determinantes sociales presentes en el resguardo indígena zenú de San Andrés de Sotavento: comunidades celeste imperio y santa elena

El Resguardo Indígena Zenú de San Andrés de Sotavento es una comunidad indígena conformada por 177 cabildos ubicados en seis municipios de los departamentos de Córdoba y Sucre, está constituido por diversas comunidades entre las que están Celeste Imperio, Santa Elena, Patio Bonito Sur, Patio Bonito Norte, Cruz del Guayabo, Las Casitas, Cruz Chiquita y Bajo Palmital. La actividad económica de los Zenúes se basa esencialmente en la agricultura, en la artesanía y en menor escala la pesca, sin embargo, aunque su economía se basa en la producción de cosechas, generan básicamente una agricultura de subsistencia. Las comunidades indígenas Zenúes, tienen una antigua tradición agrícola y una diversidad de cultivos de pan coger que respaldan sus costumbres alimenticias y su cultura ${ }^{11}$.

Los residentes en las comunidades Celeste Imperio y Santa Elena, conservan aún una identidad cultural material $^{12}$. En su tipología familiar predominan las familias extensas, que se expresan en la convivencia comunitaria, pues en un mismo predio cohabitan varias generaciones compartiendo recursos, y costumbres tanto en busca del bien colectivo, como en mejorar las posibilidades de subsistencia al sumar esfuerzos.
Determinantes intermedios: factores biológicos. La caracterización socio demográfica mostró un predominio del sexo masculino con $87 \%$, el rango de edad estuvo entre 18-70 años, con una moda de 60, el $73.4 \%$ tiene pareja sexual estable (casados ó en unión libre) con parejas elegidas entre miembros de la etnia, las familias son extensas con orientación endogámica. (Ver tabla 1 donde se ilustran los determinantes de la salud encontrados).

Determinantes intermedios: conductas y estilos de vida. En cuanto a las formas de recreación y actividad física, en las comunidades indígenas sujetos de estudio éstas no se realizan de manera programada, las actividades más afines enunciadas por los encuestados de sexo masculino fue el consumo de alcohol (cerveza) $89 \%$; en tanto que las mujeres utilizan el tiempo libre para tejer las fibras de caña flecha (65\%); en la comunidad hay ausencia de cultura del uso de elementos de protección personal en un $88 \%$.

En cuanto a la percepción de salud de los sujetos de estudio, más de la mitad (67\%) calificó su estado de salud como "regular", un $21 \%$ como "buena" y $12 \%$ respondió que "mala"; las cifras de morbilidad sentida registran que $70 \%$ de los encuestados tiene ó ha tenido alguna enfermedad en el último año y $77 \%$ presentó accidentalidad laboral.

Determinantes intermedios: circunstancias materiales, de vida y trabajo. En cuanto a este aspecto las viviendas son rústicas, carecen de servicios sanitarios como alcantarillado, las necesidades fisiológicas las realizan a campo abierto con los riesgos inherentes a la salud pública; el agua de consumo humano es recolectada de las lluvias ó de una represa cercana y consumida sin ningún tipo de tratamiento, hay convivencia con animales domésticos y proliferación de vectores. La alimentación se basa en carbohidratos y gramíneas, una vez por semana, $87 \%$ de las familias incluyen en la dieta proteína de origen animal. En lo relativo al aspecto laboral, sólo $12 \%$ emplea algún elemento de protección personal en la realización de las actividades ocupacionales.

Determinantes estructurales contexto socioeconómico y político: En el aspecto educativo se encontró que $41 \%$ tiene analfabetismo absoluto (no saben leer ni escribir y/o no ha cursado ningún año de escolaridad); $21.8 \%$ tiene primaria y bachillerato completo, sólo $3.1 \%$ de ellos culminó estudios universitarios y $1.5 \%$ lo hizo a nivel técnico. En lo concerniente a los ingresos económicos la mayorìa, 
(97\%) percibe menos de un salario mínimo mensual legal mensual vigente, muchos devengan jornales pagados a destajo. La población se caracteriza por practicar una agricultura comunitaria ${ }^{13}$, donde todos tienen derechos sobre el uso del total de las tierras asignadas: a cada familia se le adjudican dos hectáreas para su explotación y los miembro de cada familia debe aportar su fuerza de trabajo en un área de tierra para explotación comunitaria, los productos de la cosecha son distribuidos entre ellos mismos para subsistencia y comercialización de excedentes. Los ingresos económicos de estos agricultores, únicamente les permiten tener una agricultura de subsistencia, la economía de esta población es análoga a la de cualquier pueblo indígena, es decir, solidaria, de autoconsumo y de auto-subsistencia. En lo referente a seguridad social el $100 \%$ de ellos carece de afiliación a riesgos laborales y pensión, 95.3\% está afiliado al regimen de salud subsidiado. Sin embargo, es claro que no basta con tener un carnet de salud sino que es necesario saber cómo y cuándo utilizarlo, reconocer a qué se tiene derecho y tener oportunidad real de acceso a los servicios, así como recibir una atención diversificada en el contexto de su propia identidad étnica (medicina tradicional e intercultural).

Cohesión social. Las redes de apoyo comunitario son fuertes y permanentes (94\%), las familias se respaldan mutuamente mediante el intercambio de alimentos producidos en las huertas familiares (84\%), las mujeres afirman que entre ellas resuelven las situaciones que se presentan en las familias en casos de enfermedad (79\%) y muerte $(98 \%)$.

En lo concerniente a las relaciones de autoridad, el resguardo está regido administrativamente por un representante legal, el Cacique; quien ejerce gobierno sobre las comunidades que hacen parte del Resguardo incluidas las veredas con territorio indígena; cada una de estas veredas cuentan con un Capitán Mayor elegido democráticamente quien es el encargado de tomar las decisiones cuando el cacique está ausente.

Tabla 1. Determinantes sociales de salud. Resguardo indígena Zenú San Andrés de Sotavento. 2013

\begin{tabular}{|c|c|c|c|}
\hline & DETERMINANTE & COMPONENTES & $\%$ \\
\hline \multirow{7}{*}{ 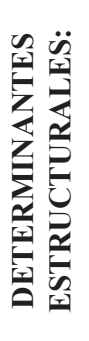 } & \multirow{7}{*}{$\begin{array}{l}\text { Contexto socio } \\
\text { económico y político }\end{array}$} & Posición socioeconómica baja: Pobreza y deprivación & 98 \\
\hline & & Economía agrícola & 100 \\
\hline & & Ingresos producto del trabajo comunitario & 93 \\
\hline & & Nivel de escolaridad: ninguna & 41 \\
\hline & & Nivel de escolaridad: primaria y bachillerato completo & 21.8 \\
\hline & & Deserción escolar para trabajo comunitario & 37.2 \\
\hline & & Gestión por líderes indígenas & 100 \\
\hline \multirow{16}{*}{ 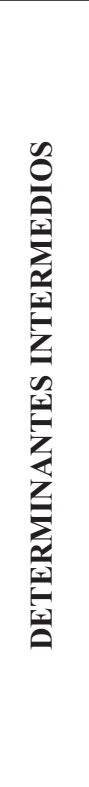 } & \multirow{6}{*}{$\begin{array}{l}\text { Circunstancias materiales } \\
\text { de vida y trabajo }\end{array}$} & Ingresos mensuales: menor de un salario mínimo legal vigente & 97 \\
\hline & & Viviendas adecuadas & 63 \\
\hline & & Ambiente físico del resguardo: insalubre & 70 \\
\hline & & Dieta balanceada & 15 \\
\hline & & Estado de salud "regular" & 67 \\
\hline & & Uso de algún elemento de protección personal en las actividades laborales & 12 \\
\hline & \multirow{3}{*}{ Seguridad social } & Afiliación a Salud: régimen subsidiado-indígena & 95.3 \\
\hline & & Afiliación a riesgos laborales & $\mathbf{0}$ \\
\hline & & Afiliación a régimen pensional: & $\mathbf{0}$ \\
\hline & \multirow{7}{*}{$\begin{array}{l}\text { Conductas, estilos de vida y } \\
\text { factores biológicos }\end{array}$} & Sexo: masculino & 87 \\
\hline & & Edad: entre 18-70 años & 100 \\
\hline & & Casados ó en unión libre con parejas elegidas entre miembros de la etnia. & 73.4 \\
\hline & & Morbilidad sentida en el último año & 70 \\
\hline & & Consumo habitual de alcohol & 73 \\
\hline & & Adultos mayores activos laboralmente & 25 \\
\hline & & Ausencia de cultura del uso de elementos de protección personal & 88 \\
\hline
\end{tabular}

Fuente: Puello Elsy, Amador Concepción y José M. Luna. Determinantes sociales de la salud de los agricultores indígenas Zenues. Córdoba. 2013 


\section{DISCUSIÓN}

Las características sociales y demográficas son elementos fundamentales en el desarrollo humano y social de una población, tal como lo postula $\operatorname{Sen}^{14}$ cuando afirma que pertenecer a una comunidad, etnia, estrato, cultura y medio social, puede afectar positiva o negativamente las opciones que tienen las personas para tener un desarrollo pleno.

En este sentido, Jaramillo ha postulado desde la antropología que en los territorios étnicos se forja "una especie de comunalismo esencialista que los aísla de realidades sociales y económicas complejas con las que interactúan las comunidades y de las que depende también su existencia" 15 situación ésta que puede dar una respuesta parcial a las condiciones encontradas entre los indígenas agricultores como son el analfabetismo, la pobreza y la escasa cobertura en seguridad social de los habitantes de las comunidades Celeste Imperio y Santa Elena (Córdoba, Colombia); condicionantes sociales que afectan en gran medida el desarrollo social de esta población y que tienen gran inherencia en la salud de estos colectivos humanos.

La poca rentabilidad de estas comunidades indígenas, se origina en las propias prácticas comunitarias que generan restricciones y limitaciones en los ingresos, pues sólo comercializan el excedente de producción agrícola porque el mayor porcentaje de la producción se reserva para la propia subsistencia comunal entre cosechas, condiciones que se reflejan en ingresos laborales paupérrimos ${ }^{16,17,18}$. Situación análoga a la que viven los pobladores de las comunidades indígenas sujetos de estudio.

La carencia de dinero originada esencialmente por la economía de subsistencia que tienen, disminuye las oportunidades para disponer de una dieta balanceada y para invertir en educación, al respecto de ésta los niños, niñas y jóvenes desertan de la escuela con el fin de aportar su fuerza de trabajo en labores comunitarias de siembra y cosecha, lo cual replica el problema social y es en este punto en donde se observa la interconexión de la baja escolaridad y la pobreza como determinantes sociales que afectan la salud en las esferas nutricionales, deficiencias de autocuidado por la escasa comprensión que le permite su nivel intelectual. Dicha cadena incesante de condicionantes sociales, más que las convicciones culturales de la etnia hace que estas comunidades indígenas se caractericen por lo básico de su modo de vida; las familias se apoyan entre sí mediante el intercambio de alimentos, de enseres y mediante la colaboración activa entre mujeres para resolver las problemáticas urgentes.

Es un derecho humano inalienable que las personas sepan leer y escribir ${ }^{19}$, en este sentido el analfabetismo encontrado en estas comunidades indígenas, refleja no sólo la ausencia de la educación como determinante de la salud, sino un fracaso en las políticas de desarrollo, y una violación de los derechos humanos. En cuanto a la educación como determinante de la salud organizaciones como CEPAL/UNESCO han declarado que las personas analfabetas tienen mayores probabilidades de siniestralidad laboral y altas tasas de morbilidad $^{19}$.

Así mismo, el hecho de saber leer, escribir ó tener algún grado de escolaridad provee al individuo de los conocimientos necesarios para desarrollar sus capacidades para vivir y trabajar con dignidad, promoviendo mejores modos de vida y por ende mejor salud ${ }^{19}$.

En esta línea de discusión, desde la óptica de la $\mathrm{UNESCO}^{20}$, la escasa o nula escolarización como la identificada en este estudio, condena a hombres y mujeres a vivir en el círculo de la pobreza por no tener las competencias necesarias para insertarse en el mercado laboral formal que les permita la obtención de trabajos decentes, mayores ingresos y mejores condiciones de vida, en este sentido, el altísimo porcentaje de analfabetismo encontrado en esta población indígena $(41 \%)$, supera con creces la media internacional, porque, según datos reportados por la la UNESCO, 796 millones de adultos, $17 \%$ de la población adulta mundial, no posee competencias básicas en lectura, escritura ni aritmética ${ }^{21}$. El grado tan alto de analfabetismo de esta población, da cuenta del estado de marginalidad y sumisión en la que se encuentran estos colectivos humanos; ubicando a los agricultores de las comunidades Celeste Imperio y Santa Helena en un plano de desigualdad frente a las personas letradas ${ }^{22}$.

En este orden, Forastieri asevera que las enfermedades y los accidentes en las personas dedicadas a labores agrícolas, tiene gran relación con su grado de educación ${ }^{23}$. Igualmente, investigadores de la línea de Escalona y Orem, plantean que la educación y el acceso a la información de los trabajadores, entre otros aspectos, son insumos críticos para la actividad humana de gran importancia en la relación salud-trabajo-enfermedad y desarrollo social ${ }^{24}$, y que juega un papel trascendental para la salud y para el auto cuidado de las personas, así como en el desarrollo de conocimiento, destrezas 
y acciones en pro de la salud de los colectivos ${ }^{25}$. Los resultados de este estudio y las discusiones realizadas en torno a este, ratifican que es trascendental, e inaplazable elevar los niveles de alfabetización y escolaridad en estas poblaciones vulnerables, de forma tal, que ello contribuya a incrementar las posibilidades de entornos saludables a nivel familiar, laboral y comunitario.

Los determinantes sociales de la salud y los Objetivos de Desarrollo del Milenio (ODM) se sustentan en la Declaración Universal de los Derechos Humanos proclamados por la Asamblea General de las Naciones Unidas el 10 de diciembre de 1948, además, la reafirman y desarrollan. En su artículo 25 la Declaración deja claro el derecho a estándares de vida adecuados para la salud y el bienestar de las personas y de sus familias, al afirmar que: "Toda persona tiene derecho a un nivel de vida adecuado que le asegure, así como a su familia, la salud y el bienestar, y en especial la alimentación, el vestido, la vivienda, la asistencia médica y los servicios sociales necesarios; tiene así mismo derecho a los seguros en caso de desempleo, enfermedad, invalidez, viudez, vejez $u$ otros casos de pérdida de sus medios de subsistencia por circunstancias independientes de su voluntad."

Como puede apreciarse, tal derecho a los estándares de vida, no aplican en ningún sentido a las comunidades indígenas estudiadas, pues si bien el Estado les ha titulado unos territorios, éste sólo le aporta alimentos básicos para su supervivencia y no les permite opciones de otros ingresos diferentes a los obtenidos con la venta de los excedentes agrícolas y de la producción artesanal con Caña Flecha; en resumen el territorio se constituye en "el soporte de las comunidad"

Los ODM han resaltado la educación primaria universal como una estrategia esencial para la reducción de la pobreza' ${ }^{9}$ en tal sentido, los indígenas de las comunidades analizadas tienen pocas oportunidades para la reducción de la pobreza, porque la relación recíproca entre salud y educación es muy clara, y en estas comunidades se encuentran factores de riesgo para la salud como la pobreza, la desnutrición y el desempleo, pero agravados por las condiciones de género, etnia y raza ${ }^{9}$.

Es reconocido que en todo el mundo, las personas socialmente desfavorecidas tienen menos acceso a los recursos sanitarios básicos y al sistema de salud en su conjunto. La falta de acceso a bienes y servicios de salud, así como la ausencia de esquemas de protección social son factores claves para explicar las inequidades, catalogadas por la OMS como injustas y evitables ${ }^{1}$.
En dichas comunidades étnicas, es clara la falta de acceso a bienes y servicios de salud, así como la ausencia de esquemas de protección social diferentes a salud (tal es el caso de pensiones y riesgos profesionales) que promueven la expansión progresiva de barreras de acceso a la salud en la tercera edad, al parecer asociados a las costumbres étnicas, culturales, de género ${ }^{9}$ y de su singular situación ocupacional. Esta asociación que ha sido analizada con suficiencia, refleja una relación de causalidad que funciona en los dos sentidos: la pobreza genera mala salud, y la mala salud hace que los pobres sigan siendo pobres ${ }^{27}$, entonces podría asumirse que el analfabetismo y la pobreza son elementos interconectados que obstaculizan a los indígenas agricultores alcanzar un óptimo estado de salud.

\section{CONCLUSIONES}

Los determinantes sociales de la salud, como se ha visto, hacen referencia al contexto social en que se desarrolla la cotidianidad humana y a a los procesos que conducen a preservar o afectar negativamente la salud. En la información analizada en las comunidades de Celeste Imperio y Santa Elena (Córdoba, Colombia) se concluye lo siguiente:

Se reflejan falencias en el contexto sociopolítico que se expresan en deficiencias en la calidad de vida producto de las inequidades en el ámbito social, económico y político, mostrando enormes fragilidades en el Sistema de Protección Social en esta población de gran vulnerabilidad. Al respecto, se identificó la baja cobertura de seguridad social con excepción de afiliación al régimen subsidiado en salud; presencia de altos índices de analfabetismo absoluto, exteriorizando baja eficiencia y efectividad del sistema educativo entre los pobladores de las comunidades; condiciones que inevitablemente afectan de manera negativa la salud de las comunidades Celeste Imperio y Santa Elena, perteneciente al Resguardo Indígena Zenú de San Andrés de Sotavento. La situación socio demográfica y de salud de estas comunidades indígenas es análoga a la de la mayoría de las poblaciones aborígenes de otros países latinoamericanos.

Las condiciones materiales en que se desarrolla la cotidianidad de la población indígena Zenú del departamento de Córdoba (Colombia) determinan serias debilidades para la subsistencia y satisfacción de las necesidades básicas producto de los bajos ingresos familiares asociados al parecer a la obligatoriedad del trabajo comunitario en los territorios asignados. Los hallazgos de esta investigación hacen visible 
la precariedad de las condiciones de vida de estos habitantes.

La comunidad indígena del Resguardo de la etnia Zenú, guarda en común la presencia de la exposición permanente e histórica relacionada con la pobreza y la desprotección relacionada con factores socioculturales como la endogamia progresiva, lo cual conduce posiblemente a la persistencia de genética con predisposición a ciertas enfermedades; sin embargo tal situación también se expresa como una fortaleza de las redes de apoyo comunitario y social identificadas por un alto grado de cohesión en la comunidad sujeto de estudio.

Entre los determinantes conductuales de la salud se observó que existe ausencia de cultura del uso de elementos de protección personal y ausencia de actividad lúdico-recreativa. Los indígenas recurren al alcohol y las indígenas a una variación de actividad laboral como diversión.

Los resultados encontrados evidencian que las circunstancias, forma y condiciones en que las viven, trabajan y envejecen los agricultores de la comunidad de indígenas del Resguardo de la etnia Zenú, su sistema de seguridad social y sus ingresos dan cuenta de su frágil situación sanitaria y su poco desarrollo social; expresada en altos índices de analfabetismo, pobreza, morbilidad sentida, accidentalidad y ausencia de cobertura en pensión y riesgos laborales, características que indiscutiblemente reafirman la relación perversa e interdependiente entre analfabetismo, pobreza y salud.

\section{RECOMENDACIONES}

Emplear los resultados de esta investigación como una herramienta que contribuya a la generación de indicadores para el desarrollo de políticas públicas socialmente responsables, para el análisis social y académico desde una perspectiva de salud intercultural, étnica y de desarrollo rural sostenible sirviendo de información para todas las personas u organizaciones que trabajan en pro de los principios de igualdad, equidad y justicia de todos los seres humanos sin distingo de raza, etnia o clase social.

La Secretaría de Asuntos Indígenas, los Ministerios de Educación, Salud y Trabajo deben analizar la frágil situación de esta comunidad étnica y establecer y/o reformular políticas públicas que disminuyan los determinantes sociales que afectan negativamente la
Salud de estos trabajadores agrícolas; encaminando estas políticas a elevar el nivel de escolaridad de la población, y aportar lineamientos que permitan el aumento de sus ingresos económicos y la cobertura de su seguridad social, de forma tal que aumenten sus posibilidades para alcanzar niveles óptimos de salud tanto para ellos como para su familia, al ampliar las posibilidades de bienestar y desarrollo social en el marco de su propia cultura.

Las diferentes organismos gubernamentales y no gubernamentales deben aumentar la inversión en educación de estos colectivos humanos, porque fomentarla, acrecentar y mejorar los niveles educativos en niños, jóvenes y adultos es una estrategia esencial para aumentar la comprensión y la capacidad para el autocuidado, tomar decisiones, analizar, participar, organizarse, gestionar alternativas para su desarrollo, superar la pobreza y aumentar las posibilidades de paz para nuestro territorio.

\section{AGRADECIMIENTOS}

Este artículo es producto de la investigación "Condiciones de trabajo, educación, salud y desarrollo social de los agricultores del Resguardo Indígena Zenú", realizada por el equipo interdisciplinario del grupo de investigación Huellas: Calidad de vida, adscrito al Departamento de Enfermería de la Universidad de Córdoba 2013.

A los indígenas agrícolas rurales, de las comunidades de Celeste Imperio y Santa Elena, dignos representantes de los diversos grupos étnicos nacionales, quienes con su sabiduría original, sus luchas y su labor han contribuido en forma definitiva a la protección del nuestro patrimonio cultural, histórico y ambiental.

Ofrecemos nuestros agradecimientos especiales a Nilvadis Arrieta, Capitana de la Comunidad Celeste Imperio de San Andrés de Sotavento y a todos los integrantes de estas comunidades indígenas por darnos la oportunidad de conocerlos, por acogernos, por su colaboración y participación en las actividades realizadas y finalmente por contribuir al enriquecimiento de nuestra vida a nivel personal y académico.

Así mismo, nuestro reconocimiento y gratitud a los enfermeros Julio Orozco Montes y José Osorio Meza, quienes como estudiantes hicieron parte de la investigación macro y desarrollaron algunos objetivos de la misma. 


\section{CONSIDERACIONES ÉTICAS}

Los aspectos éticos y legales manejados en la investigación se fundamentaron en la Resolución 008430 de 1993, la cual reglamenta la investigación en salud en Colombia. El estudio se clasificó como "sin riesgo ético", preservando la participación voluntaria y los principios de beneficencia y confidencialidad.

\section{CONFLICTO DE INTERÉS}

Los autores declaran que no existe ningún conflicto de interés en la realización de este trabajo.

\section{REFERENCIAS}

1. Organización Mundial de la Salud. Determinantes sociales de la salud. Publicación en línea. Disponible en el portal de la Organización Mundial de la Salud: http://www.who.int/social_determinants/es/.

2. Fondo Internacional de Desarrollo Agrícola. FIDA. Informe sobre la pobreza rural. Nuevas realidades, nuevos desafíos: nuevas oportunidades para la generación del mañana. Impreso por Quintily, Roma (Italia), 2011; p.68

3. Organización de las Naciones Unidas. ONU. Foro permanente para las cuestiones indígenas. Disponible en: http://social.un.org/index/indigenouses/Portada/ Cuestiones/Salud.aspx

4. Ministerio de Salud. Experiencias de instituciones educativas promotoras de salud. Perú. 2005; Disponible en: http://bvs.minsa.gob.pe/local/ MINSA/232_MINSA973.pdf.

5. Organización Mundial de la Salud. La salud de los pueblos indígenas. Centro de prensa. 2007; Nota descriptiva 326.

6. Programa de Naciones Unidas para el Desarrollo. PNUD. Informe sobre desarrollo humano de los pueblos indígenas de México. El reto de la desigualdad de oportunidades. México. 2010; p.8

7. Organización Panamericana de la Salud Organización Mundial de la Salud. Determinantes Sociales de la Salud de los Pueblos Indígenas. Quito. 2009; p. 11.

8. Melguizo, E. Cultura del cuidado de la salud. Cita a Lipson, p. 31 En Creencias y prácticas de cuidado de la salud de ancianos en situación de discapacidad y pobreza en la comuna 9 de Cartagena-Colombia. Editorial universitaria Universidad de Cartagena; 2012.

9. Tujan, A. Reducir la pobreza o edulcolar la globalización neoliberal? En: objetivos de desarrollo del milenio. 2007; p. 31, 41,42
10. Nota aclatoria: territorio colectivo, espacio geográfico dentro del territorio ancestral del Pueblo Zenú de propiedad comunitaria reconocido por el titulo colonial de 1773 y por la adjudicación legal ante la entidad estatal correspondiente (INCODER). En este territorio se ejercen leyes y gobierno propios de acuerdo a usos y costumbres.

11. Colombia. Resguardo Indígena Zenú. Cabildo Mayor Regional. Declaración del Resguardo Indígena Zenú, Córdoba y Sucre, como Territorio libre de transgénicos. San Andrés de Sotavento,2010.

12. Madera A. San Andrés de Sotavento: un pueblo Zenú. 1 ed. Bogotá D.C. Publimac. 2000; p. 55.

13. Nota aclaratoria: agricultura comunitaria, denominado también unidad económica comunitaria familiar.

14. Sen, A, Kliksberg, B. "Primero la gente. Una Mirada desde la ética del desarrollo a los principales problemas del mundo globalizado". España: Ediciones Deusto. 2007; $54 \mathrm{p}$

15. Herreño, cita a Jaramillo Efraín. La noción de territorio. 2003.

16. Mingo E, Berger M. Mundo Agrário. Vol. 10, N ${ }^{\circ}$ 19, segundo semestre. Centro de Estudios Histórico Rurales. Facultad de Humanidades y Ciencias de la Educación. Universidad Nacional de La Plata. 2009.

17. Organización de las Naciones Unidas para la Agricultura y la Alimentación. FAO. Programa Mundial de Alimentos. Informe especial. Misión FAO/PMA de evaluación de cosecha y seguridad alimentaria en Guatemala. 2010.

18. Colombia. Ministerio de la Protección Social. Gobernación de Córdoba, Departamento de Córdoba. Promoción $\mathrm{p}$ de la salud y prevención de riesgos ocupacionales en poblaciones laborales vulnerables de los municipios de Montería, Cereté, San Carlos y San Pelayo. 2007; p. 94

19. Comisión Económica para América Latina y el Caribe. CEPAL. Organización de las Naciones Unidas para la Educación, la Ciencia y la Cultura -UNESCO/ OREALC. Colección Documento de proyectos. Impacto social y económico del analfabetismo: modelo de análisis y estudio piloto. 2009; p. 5,15, 19, 47,57.

20. UNESCO. Informe de seguimiento de la educación para todos (EPT) en el mundo. Enseñanza y aprendizaje: lograr la calidad para todos. 2013 2014; p.159,160, 161,162, 170.

21. Organización de las Naciones Unidas para la Educación, la Ciencia y la Cultura. UNESCO. Una crisis encubierta: Conflictos armados y educación. Panorámica regional de América Latina y el Caribe. Informe de seguimiento de la educación para todos. 
Francia. 2011; p.5.

22. Freire P. La educación como práctica de la libertad. Uruguay: Tierra Nueva. 1970; p. 104,105

23. Forastieri, V. Programa de la OIT sobre Seguridad y Salud en la Agricultura. El reto para el siglo XXI: Prestar servicios de seguridad y salud en el trabajo a los trabajadores del agro en la revista de la OIT Educación Obrera agricultura. No 118-119, 2000; p.9,14

24. Escalona E. Relación salud-trabajo y desarrollo social: visión particular en los trabajadores de la educación. Revista Cubana de Salud Pública. P.1, 2006; 32(1):1.
25. Pastor MA, Suya L, Vásquez I, Zavala C, Ramírez T. Conocimientos y prácticas sobre autocuidado que influyen en la salud de la mujer, durante el embarazo y el puerperio. Rev Fac Cienc Méd. 2006; 13-18.

26. Herreño A. Evolución política y legal del concepto de territorio ancestral indígena en Colombia. En: El otro derecho. ํㅜ 31 -32 Agosto. ILSA. Bogotá. DC. Colombia, 2004.

27. Wagstaff A. Pobreza y desigualdades en el sector de la salud. Rev Panam Salud Publica. 2002; 11(5-6). DOI: http://dx.doi.org/10.1590/S102049892002000500007. 